\title{
Déterminants De La Fertilisation Dans Les Systèmes De Riziculture Pluviale Stricte Dans Les Zones Nord Et Sud Soudaniennes Du Burkina Faso
}

\begin{abstract}
Doctorant Abdramane Sanon,
Institut de l'Environnement et de Recherche Agricole, Laboratoire Sol-EauPlante, Station de Farako-Bâ,Burkina Faso.

Université Nazi Boni, Institut du Développement Rural, Laboratoire d'étude et de recherche sur la fertilité du sol (LERF), Bobo-Dioulasso, Burkina Faso.
\end{abstract}

\section{Alain Péoulé Kouhouyiwo Gomgnimbou,}

Institut de l'Environnement et de Recherche Agricole, Laboratoire Sol-EauPlante, Station de Farako-Bâ,, Burkina Faso.

\section{Kalifa Coulibaly,}

Université Nazi Boni, Institut du Développement Rural, Laboratoire d'étude et de recherche sur la fertilité du sol(LERF),Dioulasso, Burkina Faso.

\section{Karim Traore,}

Institut de l'Environnement et de Recherche Agricole, Laboratoire Sol-EauPlante, Station de Farako-Bâ,Bobo 01, Burkina Faso.

\section{Hassan Bismarck Nacro,}

Université Nazi Boni, Institut du Développement Rural, Laboratoire d'étude et de recherche sur la fertilité du sol (LERF), Bobo-Dioulasso, Burkina Faso

Doi:10.19044/esj.2020.v16n27p38 ～URL:http://dx.doi.org/10.19044/esj.2020.v16n27p38

\section{Résumé}

Les connaissances, les attitudes et les pratiques des agriculteurs influencent insidieusement le niveau de production rizicole. L'objectif de la présente étude est de connaître les facteurs déterminants du nombre d'apports de fumure organique, d'engrais NPK et d'urée en riziculture. Une enquête a été conduite et a concerné un échantillon de 293 riziculteurs répartis dans 14 villages, dans les régions de l'Est, des Hauts Bassins et du Plateau Central du Burkina Faso. La population enquêtée avait une moyenne d'âge de 40 ans et $37 \%$ de l'échantillon sont de sexe féminin. La méthode de la régression logistique a été utilisée pour caractériser les déterminants de la fertilisation en riziculture. Les résultats de l'étude indiquent que la moyenne d'âge est de 40 ans et 37\% de l'échantillon sont de sexe féminin. La moitié des riziculteurs enquêtés pratique les semis en lignes dans des poquets, avec un écartement de $25 * 30$ cm. Les quantités d'engrais par hectare sont faibles et sont de 398,44 kg pour 
la fumure organique, de $90,88 \mathrm{~kg}$ pour l'engrais NPK et de $56,36 \mathrm{~kg}$ pour l'engrais urée. Le riz pluvial strict est cultivé sans apport de Burkina Phosphate et de fumier de volaille. On note que l'âge du producteur, la superficie des parcelles et le nombre d'actifs agricoles sont les facteurs qui influencent le nombre d'apports de fumure organique, d'engrais NPK et d'Urée en riziculture pluviale stricte dans les zones d'étude. Dans le contexte de coût élevé des engrais minéraux, des solutions endogènes pourraient être testées dans le cadre plus large de la définition de nouvelles pratiques de fertilisation de riz pluvial strict.

Mots clef : Riz pluvial strict, Riziculteurs, Engrais, Déterminants, Burkina Faso

\section{Determinants Of Fertilization In Upland Rice Systems In The Northern And Southern Sudanian Areas Of Burkina Faso}

\section{Doctorant Abdramane Sanon,}

Institut de l'Environnement et de Recherche Agricole, Laboratoire Sol-EauPlante, Station de Farako-Bâ,Burkina Faso.

Université Nazi Boni, Institut du Développement Rural, Laboratoire d'étude et de recherche sur la fertilité du sol (LERF), Bobo-Dioulasso, Burkina Faso.

\section{Alain Péoulé Kouhouyiwo Gomgnimbou,}

Institut de l'Environnement et de Recherche Agricole, Laboratoire Sol-EauPlante, Station de Farako-Bâ,, Burkina Faso.

\section{Kalifa Coulibaly,}

Université Nazi Boni, Institut du Développement Rural, Laboratoire d'étude et de recherche sur la fertilité du sol (LERF),Dioulasso, Burkina Faso.

\section{Karim Traore,}

Institut de l'Environnement et de Recherche Agricole, Laboratoire Sol-EauPlante, Station de Farako-Bâ,Bobo 01, Burkina Faso.

\section{Hassan Bismarck Nacro,}

Université Nazi Boni, Institut du Développement Rural, Laboratoire d'étude et de recherche sur la fertilité du sol (LERF), Bobo-Dioulasso, Burkina Faso

\section{Abstract}

Knowledge, attitudes and the practices of farmers influence greatly rice productivity. The goal of this study is to identify the determiners of the 
number of organic manure and chemical fertilizers (NPK, Urea) in rice production. This study has been conducted as a survey and the sample includes 293 peasants from 14 villages in the East, Hauts bassins and Plateau Central regions of Burkina Faso. The logistic regression method was used to characterize the determinants of the value of organic manure, NPK and urea in rice farming. The results indicate that they were 40 years old in average and $37 \%$ of them women. Half of them use line pits sowing system with a spacing of $25-30 \mathrm{~cm}$. The quantity of fertilizers per hectare is less elevated with an average of $398,44 \mathrm{~kg}$ for organic manure, $90,88 \mathrm{~kg}$ for NPK and $56,36 \mathrm{~kg}$ for Urea. Upland rice is produced without using Burkina Phosphorus and poultry manure. We consider that age, the size and the number of active workers are the determiners of the value of organic manure, NPK and Urea input in upland rice production in the regions of plateau central, east and hauts bassins. With very expensive chemical fertilizers today, the contribution of the study would be the testing in agronomy of the relevance of endogenous solutions in the broad scale definition of new practices in rainy season rice production.

Keywords: Upland Rice, Farmers, Fertilizer, Value, Burkina Faso

\section{Introduction}

Le riz est un aliment de base pour plus d'un tiers de la population mondiale (Zhao et al., 2011). Au Burkina Faso, il constitue une culture stratégique et une source importante de nourriture et de revenus agricoles pour de nombreux ménages. Toutefois, moins du tiers des besoins nationaux en riz est satisfait par la production nationale, le reste étant couvert par les importations (SNDR, 2011). Paradoxalement, le pays dispose d'un potentiel important non encore exploité dans le domaine de la riziculture pluviale stricte, qui occupe seulement $15 \%$ des superficies dévolues au riz et fournit 9\% de la production nationale en riz (SNDR, 2011). Au Burkina Faso, les rendements moyens de riz pluvial strict sont inférieurs ou égaux à 1,3 t/ha (Traoré et al., 2015 ; MAAH, 2018). Les défis majeurs auxquels la riziculture pluviale stricte est confrontée, sont l'amélioration des pratiques d'apport des fumures organo-minérales et la cherté des engrais minéraux. Ces aspects ont fait l'objet de recherche par plusieurs auteurs (Gala et al., 2011 ; Traoré et al., 2015 ; Case et al., 2017 ; Xinjian et al., 2018). Des travaux sur la perception des agriculteurs en relation avec l'utilisation des fumures ont également été réalisés au niveau international (Akinwumi, 1996 ; Case et al., 2017, Xinjian et al., 2018, Hou et al., 2018). Les travaux de Case et al. (2017) ont révélé que l'incertitude quant à l'amélioration de la teneur en éléments nutritifs des sols par les engrais organiques et les difficultés de planification, constituent les principaux obstacles d'utilisation des fumures organiques. Quant aux travaux de Xinjian et al. (2018), ils ont permis d'examiner l'incidence de la perception 
des agriculteurs et de la préférence de risque des agriculteurs, sur leurs investissements en engrais organiques en Chine. En utilisant des données de plusieurs pays européens, Hou et al. (2018) ont constaté que le coût élevé et la longue période de retour sur investissement étaient les principaux obstacles à l'investissement dans les engrais organiques. En Côte d'Ivoire, l'étude de Akinwumi (1996) a montré que les principaux facteurs qui influencent de manière positive l'utilisation des engrais chimiques par les agriculteurs dans les rizières, sont la culture des bas-fonds, l'utilisation de la mécanisation, la taille de l'exploitation, la pression foncière et la disponibilité des revenus non agricoles.

Par la prise en compte des savoirs locaux, ce travail complète les études de gestion de fumures organiques et inorganiques, dans le cadre précis de la riziculture pluviale stricte. Il a pour objectif d'identifier les déterminants du nombre d'apport de fumure organique, d'engrais NPK et d'urée en riziculture pluviale stricte en zones nord et sud soudaniennes du Burkina.

\section{Matériel et méthodes \\ Milieu d'étude}

L'étude a été menée dans des villages des régions de l'Est, des Hauts Bassins et du Plateau Central du Burkina Faso (Tableau 1). Ces villages ont été considérés comme représentatifs de la diversité des conditions pédoclimatiques régionales et groupes socio-culturels ainsi que leurs pratiques en riziculture pluviale stricte.

\section{Conduite des enquêtes et support de collecte des données}

Les enquêtes ont été effectuées à l'échelle de l'Exploitation Agricole (EA) telle que défini par FAO (2000). Celle-ci représente une unité économique de production agricole, soumise à une direction unique et comprenant tous les animaux qui s'y trouvent et toute la terre utilisée entièrement ou en partie pour la production agricole, indépendamment du titre de possession, du mode juridique ou de la taille.

Ainsi, dans le cadre de notre étude, les échanges ont concerné l'exploitant qui désigne la personne physique ou morale responsable de la marche de l'exploitation agricole selon FAO (2000).

Des fiches d'enquête ont été conçues dans un premier temps en se basant sur des connaissances et des données générales déjà existantes dans les zones de l'étude.

Les principaux axes d'intérêt étaient (i) les caractéristiques socioéconomiques de l'exploitation agricole, et (ii) les perceptions paysannes des fumures organo-minérales.

Les critères de sélection des localités ont été fondés sur la représentativité, les superficies emblavées pour la riziculture pluviale stricte à partir des 
statistiques du Ministère de l'Agriculture et des Aménagements Hydroagricoles (MAAH, 2018). Ces critères avaient également servi pour la sélection des villages. Au total, quatorze (14) villages ont été sélectionnés (Tableau 1) dans quatre communes.

Tableau 1: Sites de collecte des données

\begin{tabular}{|c|c|c|c|c|}
\hline Régions & Provinces & Communes & Villages & Coordonnées \\
\hline \multirow{7}{*}{ Est } & \multirow{7}{*}{ Kompienga } & \multirow{7}{*}{ Kompienga } & Kompienga & $\begin{array}{l}11^{\circ} 44^{\prime} 34,29^{\prime \prime} \text { nord, } 0^{\circ} 45^{\prime} 20,09^{\prime \prime} \\
\text { est }\end{array}$ \\
\hline & & & Toutourgou & $\begin{array}{l}11^{\circ} 49^{\prime} 34,29^{\prime \prime} \text { nord, } 0^{\circ} 45^{\prime} 28,95^{\prime \prime} \\
\text { est }\end{array}$ \\
\hline & & & Kpankpaga & $11^{\circ} 06^{\prime} 26,9^{\prime \prime}$ nord, $0^{\circ} 06^{\prime} 27,0^{\prime \prime}$ est \\
\hline & & & Famwargou & $11^{\circ} 25^{\prime} 01^{\prime \prime}$ nord, $0^{\circ} 55^{\prime} 01^{\prime \prime}$ est \\
\hline & & & Bossoari & $11^{\circ} 1^{\prime} 26,62^{\prime \prime}$ nord, $0^{\circ} 43^{\prime} 19,62^{\prime \prime}$ est \\
\hline & & & Pognoa-Sankando & $11^{\circ} 4^{\prime} 55,09^{\prime \prime}$ nord, $0^{\circ} 35^{\prime} 02^{\prime \prime}$ est \\
\hline & & & Kpenkankanti & $11^{\circ} 4^{\prime} 54,9^{\prime \prime}$ nord, $0^{\circ} 43^{\prime} 19,67^{\prime \prime}$ est \\
\hline \multirow{2}{*}{$\begin{array}{l}\text { Hauts } \\
\text { Bassin }\end{array}$} & \multirow{2}{*}{ Houet } & Padema & Padema & $\begin{array}{l}11^{\circ} 38^{\prime} 36^{\prime \prime} \text { nord, } 04^{\circ} 15^{\prime} 41^{\prime \prime} \\
\text { ouest }\end{array}$ \\
\hline & & K-Sambla & $\begin{array}{l}\text { Soma } \\
\text { K-Sambla }\end{array}$ & $\begin{array}{l}11^{\circ} 45^{\prime} 50^{\prime \prime} \text { nord, } 04^{\circ} 09^{\prime} 52^{\prime \prime} \text { ouest } \\
11^{\circ} 13^{\prime} 13^{\prime \prime} \text { nord, } 04^{\circ} 38^{\prime} 22^{\prime \prime} \text { ouest }\end{array}$ \\
\hline $\begin{array}{l}\text { Plateau } \\
\text { Central }\end{array}$ & Ganzourgou & Boudry & $\begin{array}{l}\text { Nanom } \\
\text { Ibogo } \\
\text { Ouayalgui V5 } \\
\text { Bolma }\end{array}$ & $12^{\circ} 13^{\prime} 00^{\prime \prime}$ nord, $0^{\circ} 44^{\prime} 00^{\prime \prime}$ ouest \\
\hline
\end{tabular}

Légende : K-Sambla : Karangasso-Sambla ; Ouayalgui V5 : Aménagement de la Volta $n^{\circ} 5$ de Ouayalgui

\section{Echantillonnage des villages et exploitations agricoles}

La taille de l'échantillon d'étude a été déterminée selon la méthode de (Schwartz, 1969), basée sur le principe du tirage exhaustif suivant la formule:

$$
\mathrm{N}=\frac{Z \alpha^{2} P Q}{d^{2}}
$$

Où : $\mathrm{N}=$ Taille de l'échantillon par commune ;

$\mathrm{Z} \alpha=$ écart fixé à 1,96 qui correspond à un degré de confiance de $95 \%$;

$\mathrm{P}=$ rapport du nombre de ménages agricoles de la commune au nombre total de ménages ;

$\mathrm{Q}=1-\mathrm{P}$;

$\mathrm{d}=$ marge d'erreur qui est égale à $5 \%$

Le mode d'échantillonnage est aléatoire, pour plus d'objectivité de la valeur des estimations. La taille de l'échantillon obtenu par calcul était estimée à 286 producteurs représentant 1/3 de l'échantillon (Tableau 2). Dans l'optique de minimiser ou de garder la marge d'erreur en cas d'indisponibilité ou des données manquantes qui surviendraient dans certaines exploitations, une quinzaine de producteurs a été ajoutée. A la fin de l'enquête, huit (08) 
riziculteurs étaient indisponibles et c'est finalement 293 producteurs qui ont finalement constitué l'échantillon de l'étude.

Tableau 2: Valeurs des paramètres utilisés pour le calcul de la taille de l'échantillon

\begin{tabular}{|c|c|c|c|c|c|c|c|c|}
\hline Regions & Provinces & Communes & $\begin{array}{l}l \\
\text { Effectif } \\
\text { Producteurs } \\
\text { de riz par } \\
\text { province }\end{array}$ & $\mathbf{Z} \alpha^{2}$ & $\mathbf{Q}$ & d & $\mathbf{N}$ & $\mathrm{N} / 3$ \\
\hline Est & Kompienga & Kompienga & 13041 & 3,84 & 0,75 & 0,05 & 285 & 95 \\
\hline Hauts & & Padema & 31411 & 3,84 & 0,40 & 0,05 & 369 & 123 \\
\hline Bassins & Houet & K-Sambla & & & & & & \\
\hline $\begin{array}{l}\text { Plateau } \\
\text { Central }\end{array}$ & Ganzourgou & Boudry & 8261 & 3,84 & 0,84 & 0,05 & 202 & 67 \\
\hline Total & & & 52712 & & & & 858 & 286 \\
\hline
\end{tabular}

Légende : K-Sambla : Karangasso-Sambla ; Ouayalgui V5 : Aménagement de la Volta $\mathrm{n}^{\circ} 5$ de Ouayalgui

Source : Données de l'enquête (2019)

\section{Déroulement de l'enquête}

En prélude à l'enquête sur le terrain, des sorties exploratoires avaient permis d'avoir des informations générales sur les pratiques culturales de riz pluvial strict. Puis, un réajustement et une réadaptation des fiches ont été effectués après une phase test (Huit exploitations Agricoles par région) du questionnaire sur les différents villages en milieu rural.

\section{Traitement et analyse statistique des données}

Les données issues de l'enquête ont été codifiées puis saisies dans une matrice de gestion de base de données à l'aide du tableur Excel version 2013. Deux approches ont été adoptées pour l'analyse des données avec le logiciel SPSS (Statistical Package for the Social Sciences) version 21. Il s'agit de l'approche descriptive et de l'approche économétrique. Dans le cadre de cette dernière approche, la méthode de la régression logistique a été utilisée pour caractériser les déterminants du nombre d'apport de fumure organique, de NPK et d'urée en riziculture.

La variable dépendante dans le cadre de la régression logistique est de nature dichotomique et notée :"Nombre d'apport d'urée" : elle prend la valeur un (1) si le producteur n'applique pas de l'urée ou fait un apport unique d'urée pour la culture du riz et elle prend la valeur zéro (0) autrement.

Les variables indépendantes incluses dans la régression sont les suivantes :

- l'âge noté "âge" : existe-t-il une relation entre l'âge du riziculteur et sa décision d'apporter deux ou trois fois le NPK ou n'apporte pas d'Urée pour la culture du riz?

- la superficie notée "superf" : quelle est la relation entre la taille de la 
parcelle du riz et l'option d'appliquer deux ou trois fois le NPK, ou de ne pas appliquer l'Urée au moins une fois pour la culture du riz ?

- l'expérience du producteur notée "exp" : existe-t-il une relation entre le nombre d'années d'expérience du riziculteur et sa décision d'appliquer correctement les engrais minéraux pour la culture du riz ?

- la quantité de NPK ou d'Urée notée "NPK ou Urée" : quel lien existet-il entre les différentes modalités de cette variable, à savoir la quantité de NPK ou d'Urée, et la décision d'apporter une fois de l'engrais NPK ou deux fois l'urée pour la culture du riz?

La Corrélation a été utilisée également pour analyser les données.

\section{Résultats}

\section{Classe d'âge et niveau d'instruction des riziculteurs}

Les résultats (Tableau 3) indiquent que les femmes représentent 37\% de l'échantillon. La moyenne d'âge des riziculteurs est autour de 40 ans. Concernant le niveau d'éducation, $67 \%$ des enquêtés n'ont jamais été scolarisés, et environ $19 \%$ d'entre eux ont reçu une éducation de type formel se limitant au niveau de l'enseignement primaire et secondaire.

Tableau 3 : Statuts des exploitants agricoles

\begin{tabular}{ccc}
\hline Statut social des EA & Nombre d'EA & $\begin{array}{c}\text { Pourcentage } \\
\text { d'EA } \\
(\mathbf{n = 2 9 3 )}\end{array}$ \\
\hline Sexe & 185 & $63 \%$ \\
Masculin & 109 & $37 \%$ \\
Féminin & & \\
Age de riziculteur (an) & 79 & $27 \%$ \\
{$[20 ; 35[$} & 102 & $35 \%$ \\
{$[35 ; 50[$} & 112 & $38 \%$ \\
{$[50 ; 70[$} & & \\
Niveau d'instruction & 195 & $67 \%$ \\
Non scolarisé & 48 & $16 \%$ \\
Primaire & 9 & $3 \%$ \\
Secondaire & 24 & $8 \%$ \\
Alphabétisé & 14 & $5 \%$ \\
Non alphabétisé & 3 & $1 \%$ \\
Coranique &
\end{tabular}

Légende : EA : Exploitation Agricole

Source : Données de l'enquête (2019)

\section{Caractéristiques des exploitations agricoles}

\section{Statut économique}

Les résultats du statut économique des enquêtés sont données à travers le tableau 4 ci-dessous. Ces résultats montrent que la superficie moyenne individuelle est de 0,57 ha et que $68 \%$ des riziculteurs utilisent le petit attelage. 
Aussi, 36\% des enquêtés mènent des activités non-agricoles, notamment le petit commerce. En termes d'actifs agricoles, les résultats indiquent que $87 \%$ des riziculteurs enquêtés disposent de moins de 5 actifs agricoles hommes, et $96 \%$ des riziculteurs disposent de moins de 5 actifs agricoles femmes.

Tableau 4: Variables économiques des exploitations agricoles

\section{Statuts économiques des}

EA

\begin{tabular}{ccc}
\hline \multicolumn{3}{c}{ Superficie totale /EA (ha) } \\
{$[0,1 ; 1[$} & 269 & $91,8 \%$ \\
{$[1 ; 2[$} & 22 & $7,9 \%$ \\
{$[2 ; 3[$} & 2 & $0,7 \%$ \\
\hline Equipements agricoles & $24 \%$ \\
Manuel & 71 & $68 \%$ \\
Petit attelé & 199 & $8 \%$ \\
Gros attelé & 23 & $87 \%$ \\
\hline Nombre d'actifs agricoles hommes $(+\mathbf{1 2}$ ans $)$ \\
{$[0 ; 5[$} & 254 & $13 \%$ \\
{$[5 ; 10[$} & 37 & $1 \%$ \\
\hline $10 ; 17[$ & 2 &
\end{tabular}

Nombre d'actifs agricoles femmes (+12 ans)

\begin{tabular}{ccc}
{$[0 ; 5[$} & 281 & $96 \%$ \\
{$[5 ; 10[$} & 11 & $3 \%$ \\
{$[10 ; 12[$} & 1 & $1 \%$ \\
\hline
\end{tabular}

Activités secondaires

\begin{tabular}{ccc} 
Non & 188 & $64 \%$ \\
Oui & 105 & $36 \%$ \\
\hline
\end{tabular}

Légende : EA : Exploitation Agricole

Source : Données de l'enquête (2019)

\section{Pratiques culturales de production de riz}

Les résultats à travers le tableau 5 ci-dessous montrent que le matériel végétal se répartit en deux grands groupes à savoir les variétés améliorées (FKR45N) avec un cycle semis-maturité de 95 jours, utilisées par $87 \%$ des enquêtés, et les variétés locales de cycle semis-maturité de 95 à 120 jours, utilisées par 13\%. Aussi, les résultats montrent que 54\% des riziculteurs pratiquent les semis en poquet avec un écartement de $25 * 30 \mathrm{~cm}$ quant aux types de types de rotation, $79 \%$ des enquêtés affirment pratiquer la monoculture et seulement $6 \%$ pratiquent la rotation céréale-légumineuse (Tableau 5).

Tableau 5 : Proportion de riziculteurs par écartement entre ligne de semis et de type de rotations 


\begin{tabular}{ccc}
\hline $\begin{array}{c}\text { Ecartement } \\
\text { recommandés }\end{array}$ & Ecartement & $\begin{array}{c}\text { Pourcentage } \\
\text { d'EA (\%\%) }\end{array}$ \\
\hline \multirow{2}{*}{$25 * 25 \mathrm{~cm}$} & $30 * 30 \mathrm{~cm}$ & 27 \\
& $25 * 30 \mathrm{~cm}$ & 54 \\
& $20 * 20 \mathrm{~cm}$ & 10 \\
& $20 * 25 \mathrm{~cm}$ & 9 \\
\hline Types de & Types de & \\
rotations & rotation & \\
recommandées & Riz-Riz & 79 \\
\hline \multirow{3}{*}{ Niébé-Riz } & Maïs-Riz & 13 \\
& Niébé-Riz & 6 \\
Coton-riz & Coton-Riz & 2 \\
\hline
\end{tabular}

Légende EA : Exploitation Agricole

Source : Données de l'enquête (2019)

\section{Perceptions paysannes sur les pratiques et l'utilisation des fertilisants chimiques et organiques dans les systèmes de riziculture pluviale stricte. Opinion des paysans sur l'utilisation des fertilisants chimiques et organiques par les riziculteurs}

Les résultats à travers les tableaux 6 et 7 montrent une faible utilisation de fumure organique et minérale. $93 \%$ des enquêtés ont affirmé que l'application des engrais chimiques améliore de la fertilité des sols, et permet d'assurer de bons rendements. Malgré l'importance de cette frange des enquêtés, les données recueillies révèlent que les deux tiers des riziculteurs n'appliquent pas suffisamment les engrais minéraux. Les doses d'apport des engrais minéraux demeurent faibles sur l'ensemble des sites. En effet, ce sont en moyenne $90,88 \mathrm{~kg} / \mathrm{ha}$ pour le complexe NPK et $56,36 \mathrm{~kg} / \mathrm{ha}$ pour l'engrais urée (Tableau 7). Un tiers des riziculteurs estime que l'engrais est utilisé pour la production du maïs et du coton qui sont des cultures importantes dans leurs exploitations, le riz étant considéré comme une culture secondaire.

Selon les riziculteurs, le riz pluvial strict est généralement cultivé sans apport de Burkina Phosphate. Seulement 21,5\% de l'échantillon utilise la fumure organique pour la production de riz pluvial strict (Tableau 6), à raison de $398,44 \mathrm{~kg} / \mathrm{ha}$ par an (Tableau 7). Les types de fumures organiques les plus rencontrés sont les composts, les fumiers de bovins et d'ovins. Une minorité (moins de 1\% de riziculteurs) utilise les fientes de volaille pour la production de riz pluvial strict.

Tableau 6: Répartition des riziculteurs en fonction du nombre d'apport d'engrais en riziculture pluviale stricte

\begin{tabular}{ccccc}
\hline \multirow{2}{*}{ Nombre d'apport } & \multicolumn{3}{c}{ Pourcentage d'Exploitations Agricoles par type de fertilisant } \\
\cline { 2 - 5 } & Fumure organique & $\begin{array}{c}\text { Burkina } \\
\text { Phosphate }\end{array}$ & NPK & Urée \\
\hline Pas apport de fertilisant & $78,50 \%$ & $100 \%$ & $3,41 \%$ & $6,14 \%$ \\
\hline Apport unique & $21,50 \%$ & $0 \%$ & $89,76 \%$ & $90,44 \%$ \\
\hline
\end{tabular}


$0 \%$

$0 \%$

$6,83 \%$

$3,41 \%$

Source : Données de l'enquête (2019)

Tableau 7: Doses de fertilisants en kg/ha appliqués sur les sites en 2018.

\begin{tabular}{ccccc}
\hline Communes & Burkina Phosphate & $\begin{array}{c}\text { Fumure } \\
\text { organique }\end{array}$ & NPK & Urée \\
\hline Boudry & 0 & 0 & 74,79 & 37,77 \\
Kompienga & 0 & 975 & 100 & 100 \\
K-Sambla & 0 & 159,37 & 86,83 & 40,93 \\
Padema & 0 & 60,96 & 101,91 & 46,75 \\
\hline Moyenne générale & $\mathbf{0}$ & $\mathbf{3 9 8 , 4 4}$ & $\mathbf{9 0 , 8 8}$ & $\mathbf{5 6 , 3 6}$ \\
\hline
\end{tabular}

Légende EA : K-Sambla : Karangasso-Sambla

Source : Données de l'enquête (2019)

\section{Déterminants de la fertilisation organo-minérale}

Les tableaux 8 et 9 présentent les résultats sur les déterminants de la fertilisation organo-minérale. Le tableau 10 présent la matrice de corrélation entre les différents paramètres. Les résultats ont montré que le modèle de régression logistique est valide avec un pouvoir explicatif d'environ $80 \%$ (Tableau 8).

Age: On note une corrélation positive $(\mathrm{r}=0,064)$ mais non significative entre l'âge et l'apport unique d'urée. Le nombre d'apport de l'urée est fonction de l'âge. En effet, en cas d'apport d'urée, les personnes âgées le font en une seule fois. La probabilité d'utilisation d'apport fractionné de l'urée est susceptible d'augmenter de 0,64 fois.

Superficie: Les résultats indiquent une corrélation négative $(\mathrm{r}=$ $0,067)$ non significative entre la superficie et l'apport non fractionné d'urée. En effet, plus les superficies sont de petites tailles, plus les producteurs appliquent deux ou trois fois l'Urée. Le coefficient de régression logistique reflète que le nombre d'apports d'urée est probable d'augmenter de 0,288 fois avec la taille de la superficie des parcelles.

Expérience : On note que l'expérience du riziculteur n'est pas corrélée au fait d'apporter une fois l'urée ou de ne pas l'apporter. En revanche les résultats montrent une relation positive et significative $(\mathrm{r}=, 144)$ entre l'apport fractionné d'urée et l'expérience du riziculteur. Cela indique que les riziculteurs expérimentés sont plus susceptibles de fractionner l'urée que les moins expérimentés. Le nombre d'apport fractionné de l'urée est susceptible d'être multiplié par 0,047.

Nombre d'actifs : Il existe une relation positive $(\mathrm{r}=0,07)$ mais non significative entre le nombre d'actif et la décision de ne pas fractionner l'urée. En effet, plus les producteurs ont des actifs, plus ils sont enclins à l'apport fractionné de l'urée. L'application fractionnée de l'urée est susceptible d'augmenter de 0,085 fois avec le nombre d'actif. 
Nombre d'apport de Fumure organique : Le nombre d'apport d'urée n'est pas corrélée $(\mathrm{r}=-0,191)$ au fait d'apporter une fois la fumure organique. Plus l'urée est fractionnée plus on assiste à une réduction du nombre d'apport de fumure organique. Le nombre d'apport de fumure organique est susceptible de diminuer de 0,183 fois avec le fractionnement d'urée.

Quantité de FO, d'urée et de NPK : les résultats ont montré qu'il n'existe pas de lien entre la quantité de FO, d'Urée, de NPK disponible dans l'exploitation et la décision de ne pas fractionner l'urée. Cela indique que les riziculteurs qui disposent d'une quantité d'Urée, de NPK et de FO, sont enclins au fractionnement d'urée.

Tableau 8 : Evaluation de l'ajustement du modèle

\begin{tabular}{ccccc}
\hline Individus & Log Vrais. & L.R. Khi-2 & Pr. $>$ L.R. Khi & R $^{\mathbf{2}}$ \\
\hline 293 & $-12,404$ & 110,495 & $<0,0001$ & 0,8 \\
\hline
\end{tabular}

Tableau 9 : Valeurs estimées des paramètres du modèle du nombre d'apport d'engrais Urée associé à l'engrais NPK en combinaison avec la Fumure Organique

\begin{tabular}{ccccc}
\hline Paramètre & Valeur estimée & Ecart-type & Khi $^{\mathbf{2}}$ & Pr. $>\mathbf{K h i}^{\mathbf{2}}$ \\
\hline Constante & 17,505 & 8,215 & 4,541 & 0,033 \\
Age & $-0,042$ & 0,091 & 0,207 & 0,649 \\
Sup & $-4,707$ & 4,428 & 1,130 & 0,288 \\
EXP & $-2,635$ & 1,325 & 3,954 & 0,047 \\
N_actifs & $-0,759$ & 0,441 & 2,959 & 0,085 \\
Qté FO (kg/ha) & $-0,006$ & 0,005 & 1,801 & 0,180 \\
N_FO & $-1,125$ & 0,846 & 1,770 & 0,183 \\
Qté de NPK (Kg/ha) & 0,062 & 0,031 & 4,009 & 0,045 \\
N_NPK & $-7,394$ & 4,238 & 3,044 & 0,081 \\
Qté d'Urée (kg/ha) & $-0,205$ & 0,073 & 7,797 & 0,005 \\
Rd (kg/ha) & 0,000 & 0,000 & 0,711 & 0,399 \\
\hline
\end{tabular}

Légende : N_NPK : Nombre d'apport de NPK; Qtité de NPK : Quantité de NPK ; EXP : Expérience ;

$\boldsymbol{N} \_$actif : Nombre d'actifs, $\boldsymbol{R} \boldsymbol{d}:$ Rendement ; $\boldsymbol{N}_{\mathbf{F}} \boldsymbol{F \boldsymbol { O }}:$ Nombre d'apport de Fumure organique;

N_Urée : Nombre d'apport d'urée ; Qtité d'Urée : Quantité d'Urée ; Sup : Superficie.

Tableau 10 : Corrélation de Pearson entre les différents paramètres

\begin{tabular}{|c|c|c|c|c|c|c|c|c|c|c|c|}
\hline Corrélations & AGE & & EXP & N_actifs & N_FO & Qté FO & N_NPK & $\begin{array}{c}\text { Qté } \\
\text { NP } \\
\text { K }\end{array}$ & $\begin{array}{l}\text { Qté } \\
\text { Urée }\end{array}$ & $\begin{array}{c}\mathbf{N}_{-} \\
\text {Uré } \\
\text { e }\end{array}$ & $\begin{array}{l}\mathbf{R} \\
\mathbf{d}\end{array}$ \\
\hline AGE & 1 & & & & & & & & & & \\
\hline EXP & 0,069 & 1 & & & & & & & & & \\
\hline Sup1 & ,142* & 0,066 & 1 & & & & & & & & \\
\hline N_actifs & $\begin{array}{c}, 446^{*} \\
*\end{array}$ & 0,024 &, $267 * *$ & 1 & & & & & & & \\
\hline N_FO & $0, \overline{0} 84$ &,$- 242 * *$ &,$- 233 * *$ &,$- 136^{*}$ & 1 & & & & & & \\
\hline
\end{tabular}




\begin{tabular}{|c|c|c|c|c|c|c|c|c|c|c|c|}
\hline Qté FO & $\begin{array}{c}, 181^{*} \\
*\end{array}$ &, $305 * *$ &, $637 * *$ & 0,089 &,$- 342 * *$ & 1 & & & & & \\
\hline N_NPK & $\begin{array}{c}- \\
0,047\end{array}$ & 0,056 & $-0,006$ & 0,038 & 0,09 & $-0,094$ & 1 & & & & \\
\hline Qté NPK & 0,052 & ,131* &,$- 130^{*}$ & 0,006 & $-0,017$ & 0,097 & 0,085 & 1 & & & \\
\hline Qté Urée & $0, \overline{0}-6$ & 0,097 &,$- 129 *$ & $-0,003$ & $-0,031$ & 0,086 & 0,053 &, $720 * *$ & 1 & & \\
\hline N_Urée & 0,064 & ,144* & $-0,067$ & 0,07 &,$- 191^{* *}$ & 0,063 & 0,026 & 0,039 & 0,02 & 1 & \\
\hline Rd & $\begin{array}{c}, 205^{*} \\
*\end{array}$ & ,128* &, $169^{* *}$ & 0,096 &,$- 366^{* *}$ &, $278^{* *}$ & $-0,036$ & 0,079 & $\begin{array}{c}0,04 \\
4\end{array}$ & 0,029 & 1 \\
\hline
\end{tabular}

* La corrélation est significative au niveau 0,05 (bilatéral).

** La corrélation est significative au niveau 0,01(bilatéral).

Légende : N_NPK : Nombre d'apport de NPK ; Qtité de NPK : Quantité de NPK ; EXP :

Expérience ;

N_actifs : Nombre d'actifs, Rd : Rendement ; N_FO : Nombre d'apport de Fumure organique ;

N_Urée : Nombre d'apport d'urée ; Qtité d'Urée : Quantité d'Urée ; Sup : Superficie.

\section{Discussion}

\section{Pratiques culturales dans les systèmes de riziculture pluviale stricte}

L'étude montre que la superficie moyenne des parcelles est de 0,57 ha. Les semences de variétés améliorées sont les plus utilisées. Les mêmes constats ont été faits par Traore et al. (2015). En milieu paysan, la plupart des riziculteurs qui commercialisent le riz, produisent le riz pluvial en utilisant des variétés améliorées de riz pluvial (Gala, 2009). L'utilisation de ces semences de variétés permet d'améliorer la production et les revenus des exploitants, en particulier dans un contexte de changement climatique. Toute chose qui permettra de réduire l'importation du riz.

Cependant, les résultats ont montré une faible utilisation de la fumure organo-minérale par la majeure partie des enquêtés. Des résultats similaires ont été rapportés par des travaux antérieurs dans les systèmes de production à base de riz pluvial strict dans les exploitations agricoles en Afrique de l'Ouest (Manyong et al., 2001 ; Traoré et al., 2015). Ceci est dû aux recommandations généralisées pour de vastes zones, qui ne tiennent pas compte de la complexité et de la diversité des contextes d'exploitation des agriculteurs (Oikeh et al., 2015). Dans le cadre de cette étude, les riziculteurs estiment que les coûts élevés des engrais minéraux et leurs faibles disponibilités sur les marchés locaux expliquent cette faible utilisation d'engrais chimiques. Cet état de fait a été déjà rapporté par Xu et al. (2014) en Chine et par Gala et al. (2011) en Côte d'Ivoire. Du fait de la faible disponibilité de la fumure organique, celleci est utilisée prioritairement pour la production du coton et du maïs, qui sont des cultures plus importantes et destinées à la commercialisation; le riz n'est qu'une culture secondaire.

Les effets perçus d'augmentation du rendement et d'amélioration de la qualité de la fertilité des sols, encouragent les producteurs à investir dans les fumures organiques ; toutefois, l'augmentation des charges liées aux coûts de 
production, les découragent. Une autre raison serait que, lorsque les fortes doses des fertilisants sont utilisées, l'augmentation de rendement qu'elles entrainent ne fait que compenser les dépenses engagées pour leur achat, et n'engendre aucun profit (Kaho et al., 2011 ; Mucheru et al., 2007).

Par ailleurs, les engrais chimiques jouent un rôle incontournable dans la production agricole (Bationo et Somda, 1994), mais leur coût élevé constitue un frein à leur adoption (Gomgnimbou et al., 2019 ; Randrianarisoa et Minten, 2003 ; Minten et Ralison, 2003). Nos résultats sont conformes à ceux de Gala et al. (2007) qui ont montré que compte tenu du coût élevé et de la faiblesse du pouvoir d'achat de la population rurale, les engrais ne sont pas effectivement utilisés par les producteurs de riz pluvial strict de la Côte d'Ivoire. Dans les régions de l'Est, des Hauts Bassins, et du Plateau Central du Burkina Faso, le coût élevé des engrais minéraux et leur faible disponibilité sur les marchés locaux, emmènent certains riziculteurs à apporter l'engrais NPK deux ou trois fois pour combler le déficit en Urée. Les mêmes observations ont été faites par Xinjian et al. (2018) en Chine, et par Hou et al. (2018) en Europe. Les principaux obstacles à l'adoption d'une technologie, sont liés à des facteurs économiques. En outre pour notre cas, la faible disponibilité de l'eau limite significativement la production de fumure organique, et donc son utilisation en riziculture pluviale stricte.

Contrairement aux recommandations faites pas les structures de recherche, seule une faible proportion de riziculteurs apportent les engrais minéraux de façon fractionnée (6,83\% pour le NPK et 3,41\% pour l'urée). Si les riziculteurs sont attachés aux pratiques anciennes, traditionnelles à caractère ancestral et transmises de manière générationnelle (Gala et al., 2007 ; Akpo et al., 2016), il faut aussi admettre, en tout cas pour la présente étude, que le manque de formation sur les modes d'application des engrais chimiques, l'absence d'information et la méconnaissance, expliquent les insuffisances observées et la non utilisation du Burkina Phosphate en riziculture pluviale stricte. Le riz est cultivé en continue par la majorité des producteurs enquêtés, même si quelques cas de rotations maïs-riz et niébé-riz ont été rencontrés. Ce résultat conforte l'argument de Traoré et al. (2015) selon lequel le riz pluvial strict est cultivé en pure sans rotation régulière.

\section{Modèle de régression logistique}

La cherté des engrais minéraux de leur faible disponibilité sur le marché local emmène certains riziculteurs à ne pas apporter de l'urée ou faire un apport unique d'urée en riziculture pluvial stricte. D'après les résultats de l'analyse descriptive et de la régression logistique, les riziculteurs qui sont prêts à adopter cette pratique de fertilisation sont constitués essentiellement de personnes âgées ayant de grande superficie de riz avec peu d'actifs agricole. Selon Gala (2009) en riziculture pluviale de plateau dans la zone forestière de 
la Côte d'Ivoire, d'un côté, la cherté des engrais chimiques ne permet pas aux producteurs de riz pluvial strict d'utiliser les doses conseillées, de l'autre, le manque de connaissance du mode d'application se révèle à travers diverses modalités de fractionnement des engrais minéraux, notamment l'urée. Le même constat a été fait sur les sites de notre étude au Burkina Faso. En faisant un apport unique d'urée pour la culture du riz, ces riziculteurs n'ont d'autres alternatives que d'apporter de grande quantité de NPK et de fumure organique. Les résultats de la régression montrent que les riziculteurs ayant une quantité importante d'engrais NPK dans leurs exploitations, produisent le riz pluvial strict sans apport d'urée. Pour compenser le manque d'urée, il faut nécessairement un apport important d'engrais minéraux NPK et de fumure organique, tel que constaté chez certains de nos enquêtés. Ces résultats confirment les études de Lamb et al. (2003) qui ont démontré que selon les agriculteurs des pays en développement, l'incertitude de la production peut conduire à une utilisation excessive d'engrais minéraux. L'apport excessif d'engrais inorganiques et organiques par les riziculteurs est conforme aux constats faits antérieurement en productions maraichères par Ouikoun et al. (2019). Les résultats de l'étude ont également montré que plus les riziculteurs sont expérimentés avec beaucoup actifs, plus ils font des fractionnements d'urée. En effet, lorsque le nombre d'actifs agricole est important dans l'exploitation agricole, il permet aux exploitants d'utiliser cette main d'œuvre pour les travaux d'entretien des parcelles notamment l'application des engrais. C'est pourquoi le nombre d'actif a un effet positif sur les nombres d'apport d'urée. Dans une exploitation ayant une grande superficie de riz, c'est donc le nombre d'actif qui explique le fractionnement d'urée plutôt que le prix et la disponibilité de cet engrais.

\section{Conclusion}

Ce travail avait pour objectif d'identifier les déterminants du nombre d'apport de fumure organique, de NPK et d'urée en riziculture pluviale stricte dans les régions de l'Est, du Plateau Central et des Hauts Bassins du Burkina. Cette étude a montré que plus de la moitié des riziculteurs enquêtés connaissent le rôle des fumures organo-minérales dans l'obtention de bons rendements du riz pluvial strict. La majorité des exploitants rencontrés pratique un fractionnement de l'engrais NPK et de fumure organique. Les producteurs âgés, avec peu d'actifs agricoles ayant des superficies relativement importantes ne procèdent pas au fractionnement d'urée. Et plus les producteurs ont de l'expérience dans la production de riz pluvial strict, plus ils sont enclins à un apport fractionnement d'urée en associant l'engrais NPK et organique. Les riziculteurs les moins âgés ayant des superficies de riz de petites tailles font le fractionnement de NPK et d'engrais organique en combinaison avec l'apport fractionné d'Urée. Les résultats suggèrent qu'il faut 
promouvoir l'intensification de la production rizicole comme moyen d'utilisation de la fumure organo-minérale dans les zones nord et sud soudaniennes du Burkina Faso. Les groupes cibles doivent être identifiés en fonction des déterminants du choix de leur mode de gestion de la fertilité du sol en riziculture pluviale stricte : producteurs âgés avec superficies emblavées relativement importantes mais peu d'actifs agricoles, producteurs expérimentés, producteurs moins âgés avec des superficies petites tailles. Au regard de l'insuffisance dans la pratique de fertilisation et de l'absence d'utilisation du Burkina Phosphate en riziculture pluviale stricte, il est nécessaire d'élaborer des recommandations sur les engrais «à la carte » et de renforcer la compréhension des agriculteurs en matière d'engrais minéraux et d'utilisation de Burkina Phosphate. L'apport de la recherche pourrait consister à tester la validité et la solidité des solutions endogènes, notamment celles relatives au fractionnement des engrais complexes (NPK) et des fumures organiques (Fumier de volaille) riche en azote en riziculture pluviale stricte.

\section{References:}

1. Akinwumi A.A. (1996). Factors affecting the adoption of fertilizers by rice farmers in Côte d'Ivoire. Nutrient Cycling in Agroecosystems, $46: 29-39$.

2. Akpo M.A.A., Saidou I, Balogoun Y.I., Bio BL. B. (2016). Evaluation de la performance des pratiques de gestion de la fertilité des sols dans le bassin de la rivière Okpara Au Benin. European Scientific Journal, 12 (33) :1857 - 7881.

3. Bationo A., Somda Z. (1994). Gestion de la fertilité des sols. Le travail du sol pour une agriculture durable, Cours de formation 4 13 juillet 1994. FAO, W.B. Hoogmoed M.C. Klaij. Niamey, Niger. www.fao.org. $21 \mathrm{p}$.

4. Case S., Oelofse M., Hou Y., Oenema O., Jensen L.S. (2017). Farmer perceptions and use of organic waste products as fertilisers. A survey study of potential benefits and barriers. Agric. Syst., 151, 84-95.

5. FAO. (2000). Programme du recensement mondial de l'agriculture 2000. Collection FAO: Développement statistique numéro 5, FAO, Rome, 1995, 28 p.

6. Gala J.T.B., Camara M., Assa A., Keli J.Z. (2007). Problématique de l'utilisation des Engrais Minéraux dans les zones de production du riz : Cas du Centre-Ouest de la Côte D'ivoire. Agronomie Africaine, 19 (2) : 173-185.

7. Gala J.B.T. (2009). Optimisation de la fertilisation en riziculture pluviale de plateau en Côte d'Ivoire : cas de la zone forestière semi- 
décidue. Thèse de doctorat ; Université de Cocody, Abidjan, Côte d'Ivoire, $154 \mathrm{p}$.

8. Gala T.J., Camara M., Yao-Kouame A., Keli Z. J. (2011). Rentabilité des engrais minéraux en riziculture pluviale de plateau : Cas de la zone de Gagnoa dans le centre ouest de la Côte d'Ivoire. J. Appl. Biosci. 46, : 3153-3162.

9. Gomgnimbou A.P.K., Bandaogo A.A., Coulibaly K., Sanon A., Ouattara S. et Nacro H.B. (2019). Effets à court terme de l'application des fientes de volaille sur le rendement du maïs (Zea mays L.) et les caractéristiques chimiques d'un sol ferralitique dans la zone sud-soudanienne du Burkina Faso. International Journal of Biological and Chemical Sciences, 13 (4) : 2041-2052.

10. Hou Y. Velthof G.L., Case S.D.C., Oelofse M., Grignani C., Balsari P., Zavattaro L., Gioelli F., Bernal M.P., Fangueiro D. (2018). Stakeholder perceptions of manure treatment technologies in Denmark, Italy, The Netherlands and Spain. J. Clean. Prod., 172 :1620-1630.

11. Lamb R.L. (2003). Fertilizer use, risk, and off-farm labor markets in the semi-arid tropics of India. Am. J. Agric. Econ., 85 : 359-371.

12. MAAH (Ministère de l'Agriculture et des Aménagements Hydrauliques). (2018). Annuaire des statistiques agricoles 2017. Direction Générales des Etudes et des statistiques Sectorielles, Ministère de l'Agriculture et des Aménagements Hydro-agricoles, Ouagadougou, Burkina Faso, 197 p.

13. MFSNF (Ministere de la Femme, de la Solidarite Nationale et de la Famille). (2016). Annuaire Statistique « Femme et Genre » 2016. Ministere de la Femme, de la Solidarite Nationale et de la Famille, Ouagadougou, Burkina Faso, 64 p.

14. Manyong V.M., Makinde K.O., Sanginga N., Vanlauwe B., Diels B. (2001). Fertilizer use and definition of farmer domains for impactoriented research in northern guinea savannah of Nigeria. Nutrient Cycling in Agroecosystems, 59: 129-141.

15. Minten B. et Ralison E. (2003). Durabilité de l'environnement, agriculture et pauvreté à Madagascar. Conférence «Agriculture et Pauvreté », 20 mars 2003, Antananarivo. FOFIFA. 14 p.

16. Oikeh S.O., Nwilene F., Diatta S., Osiname O., Touré., A., Okeleye K.A. (2008). Responses of Upland NERICA Rice to Nitrogen and Phosphorus in Forest Agroecosystems. Agronomy Journal, 100 : 735-741.

17. Ouikoun G.C., Bouka C. E., Lawson-Evi P., Dossou J., EkluGadégbeku K. (2019) Caractérisation des Systèmes de Cultures des 
Sites Maraîchers de Houéyiho, de Sèmè-Kpodji et de Grand-Popo au Sud-Bénin. European Scientific Journal Vol.15, No.18.

18. Randrianarisoa J.C., Minten B. (2003). Accessibilité et utilisation des engrais chimiques à Madagascar, Conférence "Agriculture et pauvreté". FOFIFA-CORNELL-CNRADR-Programme ILO, Antanarivo, $6 \mathrm{p}$.

19. Schwartz D. (1969). In : Méthodes Statistiques à l'Usage des Médecins et les Biologistes. $3^{\mathrm{e}}$ edn., Flammarion Médecine Sciences, Paris, p. 318

20. SNDR (Stratégie nationale de développement de la riziculture). (2011). Ministère de

l'Agriculture, de l'Hydraulique et des Ressources Halieutiques, Burkina Faso, 43 p.

21. Traoré A., Traoré K., Traoré O., Bado V.B., Nacro H.B., Sedogo P.M. (2015). Caractérisation des systèmes de production à base de riz pluvial strict dans les exploitations agricoles de la zone Sudsoudanienne du Burkina Faso. Int. J. Biol. Chem. Sci., 9 (6) : 26852697.

22. Xinjian C., Di Z., Ying X., Xiao J.F. (2018). Perceptions, Risk Attitude and Organic Fertilizer Investment: Evidence from Rice and Banana Farmers in Guangxi, China. Sustainability, 10, 3715.

23. Xu H., Huang X., Zhong T., Chen Z., and Yu J. (2014). Chinese land policies and farmers'adoption of organicf ertilizer for saline soils. Land Use Policy, 38 : 541-549.

24. Zhao J., Ni T., Li J., Lu Q., Fang Z., Huang Q., Zhang R., Li R., Shen B., Shen Q. (2016). Effects of organic-inorganic compound fertilizer with reduced chemical fertilizer application on crop yields, soil biological activity and bacterial community structure in a ricewheat cropping system. Appl. Soil Ecol., 99 : 1-12.

25. Zhao L., L. Wu, M., Li. Y. (2011). Nutrient uptake and water use effiency as effected by modified rice cultivation methods with irrigation. Paddy water environ Journal, 9:25-32. 